Quim. Nova, Vol. 36, No. 6, 874-879, 2013

\title{
ANÁLISE EXPLORATÓRIA RÁPIDA E NÃO DESTRUTIVA (SCREENING) DA PRESENÇA DE ELEMENTOS QUÍMICOS TÓXICOS EM MATERIAL ESCOLAR POR FLUORESCÊNCIA DE RAIOS X
}

\author{
José Augusto Da-Col, Rafael Ortiz Sanchez, Juliana Terra e Maria Izabel Maretti Silveira Bueno* \\ Instituto de Química, Universidade Estadual de Campinas, CP 6154, 13084-971 Campinas - SP, Brasil
}

Recebido em 5/7/12; aceito em 30/11/12; publicado na web em 8/3/13

\begin{abstract}
SCREENING INVESTIGATION OF TOXIC CHEMICAL ELEMENTS IN SCHOOL SUPPLIES BY X-RAY FLUORESCENCE. Compulsory in Brazil, toy certification aims to prevent possible risks in toy use. The Brazilian National Institute of Metrology (INMETRO) establishes the maximum concentration of toxic elements (TE) that may be present in toys. This study evaluates the presence of TE in different crayons, gouache and modeling clays using X-ray fluorescence. This technique is fast, has low operating cost and minimum sample pretreatment, resulting in a clean procedure without reagent consumption and waste generation. Bromine (in gouache) and Barium (in crayons) were the only potentially TE identified in the samples studied.
\end{abstract}

Keywords: school supplies; screening; X-ray fluorescence.

\section{INTRODUÇÃO}

Produtos como massa de modelar, tinta guache e giz de cera não são apenas empregados como material escolar, mas também são utilizados em atividades por crianças que ainda não atingiram a idade escolar. Nesta fase da vida, devem ser redobrados os cuidados com possíveis contaminações por elementos químicos tóxicos, pois podem ocorrer problemas sérios e irreversíveis. ${ }^{1,2}$

Alimentos e água contaminados, assim como objetos que possam ser levados à boca ou ingeridos, são fontes expressivas de contaminação de metais tóxicos. ${ }^{3,4}$ Um exemplo é o relato da intoxicação de uma criança de 4 anos após a ingestão de uma medalha, cuja análise da composição indica presença de $38 \%$ de $\mathrm{Pb}$ (em massa), o que elevou a concentração deste metal no sangue da paciente em 12 vezes acima do valor considerado normal. ${ }^{5} \mathrm{Na}$ Índia, brinquedos de poli(cloreto de vinila), PVC, apresentaram teores de $\mathrm{Cd}$ e $\mathrm{Pb}$ acima dos limites indicados pela Agência de Proteção Ambiental do governo norte americano; a eventual lixiviação desses metais tóxicos pode oferecer risco às crianças. ${ }^{6}$

No Brasil, em 2009, foi realizado um estudo com brinquedos adquiridos na fronteira Brasil-Paraguai e no comércio popular de São Paulo e, através de técnicas como fluorescência de raios $\mathrm{X}$ (XRF), ativação neutrônica (INAA) e cromatografia gasosa (GC) foi comprovada a contaminação de algumas amostras por $\mathrm{Ba}, \mathrm{Pb}$ e Th, além do composto orgânico dibutilftalato. ${ }^{7}$ No mesmo ano, pesquisadores detectaram teores preocupantes de $\mathrm{Ni}$, Co e $\mathrm{Cr}$ em kits de maquiagem infantil através do uso da espectrometria de absorção atômica com forno de grafite (GFAAS) e digestão assistida por radiação micro-ondas. ${ }^{8}$

Godoi et al. determinaram $\mathrm{Ba}, \mathrm{Cd}, \mathrm{Cr}$ e $\mathrm{Pb}$ em carrinhos e blocos de montar plásticos adquiridos no mercado informal. As amostras foram analisadas com a técnica de espectrometria de emissão óptica com plasma induzido por laser (LIBS) e os teores dos metais foram compatíveis com aqueles determinados por espectrometria de emissão óptica com plasma indutivamente acoplado (ICP OES). Para afirmar que as amostras poderiam representar um risco, a norma ABNT NBR NM 300, regulamentada em 2005 pelo Instituto Nacional de Metrologia, Normalização e Qualidade Industrial (INMETRO) foi usada como referência. ${ }^{9}$ Essa norma torna obrigatória a certificação

\footnotetext{
*e-mail: bell@iqm.unicamp.br
}

de todo brinquedo comercializado no Brasil, independente de ter sido fabricado no país. ${ }^{10}$

A certificação de brinquedos tem como intenção evitar eventuais riscos que possam surgir pelo uso normal ou indevido do brinquedo. Os principais ensaios realizados são os de impacto, queda, mordida, tração, composição química, inflamabilidade e ruído. ${ }^{10}$ Atendendo aos requisitos da legislação, os fabricantes recebem o Certificado de Conformidade e podem utilizar o selo de qualidade do INMETRO nos produtos.

Com relação à composição, os ensaios químicos visam identificar e quantificar elementos químicos nocivos à saúde. A norma estabelece, por exemplo, limites máximos de elementos químicos tóxicos que possam estar presentes em brinquedos (Tabela 1). ${ }^{11}$

Tabela 1. Concentração máxima permitida pela norma ABNT NBR NM 300 para elementos químicos potencialmente tóxicos em brinquedos (g/100 g)

\begin{tabular}{cc}
\hline Elemento & Valor máximo permitido \\
\hline Antimônio & 0,0060 \\
Arsênio & 0,0025 \\
Bário & 0,1000 \\
Cádmio & 0,0075 \\
Chumbo & 0,0090 \\
Cromo & 0,0060 \\
Mercúrio & 0,0060 \\
Selênio & 0,0500 \\
\hline
\end{tabular}

Para fins legais, o INMETRO define, no item 3.52 da norma NM $300 / 2002$, que brinquedo é "qualquer material projetado, ou claramente destinado, para o uso de brincadeiras por crianças menores de 14 anos de idade". Essa definição é próxima daquela encontrada na portaria 188/2007 para materiais escolares: "quaisquer objetos ou materiais projetados para uso por crianças menores de 14 anos em atividades educativas em grupos ou individuais". Essa similitude pode causar ambiguidade na interpretação da norma NM 300/2002 para itens como massa de modelar, giz de cera e guache que, além de serem utilizados em atividades escolares, devido a aplicações didáticas e pedagógicas, também podem ser classificados como brinquedos. Essa dupla aplicação poderia fazer com que os ensaios de segurança 
não fossem realizados, já que materiais escolares ficariam isentos de certificação nesta norma. ${ }^{11}$

Por outro lado, recentemente, o INMETRO, juntamente com o Ministério do Desenvolvimento, Indústria e Comércio Exterior, publicou uma portaria alertando que a certificação de materiais escolares no país passará a ser compulsória. ${ }^{12}$

No processo de fabricação de brinquedos e materiais escolares, são acrescentados vários aditivos às matrizes, que podem conter elementos químicos tóxicos, causando intoxicação às crianças em caso de ingestão "acidental" do produto ou parte dele.

Neste estudo, foi empregada a fluorescência de raios X (XRF), que é uma técnica de análise relacionada com a medida de energia e intensidade características da radiação X emitida por uma amostra irradiada com partículas ou fótons de alta energia. ${ }^{13}$ Para elementos químicos classificados como pesados $(Z>11)$, a energia liberada no decaimento eletrônico dos átomos da amostra irradiada, após ocorrer o efeito fotoelétrico, permite obter informações qualitativas da amostra, pois nesse processo cada elemento químico libera uma energia característica. E, pelo fato das intensidades das energias emitidas serem proporcionais às quantidades dos elementos químicos na amostra, suas concentrações podem ser determinadas. ${ }^{14}$ Assim, a XRF pode ser aplicada na resolução de vários problemas analíticos, seja em medicina, ${ }^{15}$ geologia, ${ }^{16}$ biologia, ${ }^{17}$ arqueologia ${ }^{18}$ ou monitoramento ambiental. ${ }^{19}$

Uma característica intrínseca das análises usando XRF é a interferência eventual causada pelo efeito interelementar que, em amostras muito complexas, pode promover a diminuição ou intensificação de um sinal, resultando em um valor errôneo de concentração. Uma ferramenta matemática que pode ser utilizada para minimizar esse problema é o método de Parâmetros Fundamentais (PF), método esse que, por poder não utilizar padrões para comparação, é considerado como absoluto. Esse método é baseado em cálculos matemáticos iterativos que correlacionam a intensidade de uma dada linha de emissão com a concentração do elemento fluorescente. ${ }^{20}$ Programas computacionais que efetuam esta quantificação são, em geral, disponibilizados pelos fabricantes de equipamentos de fluorescência de raios X.

Ferramentas quimiométricas, como a Análise de Componentes Principais (PCA, Principal Component Analysis), podem ser empregadas para se realizar a análise exploratória de amostras por XRF. A PCA reduz a dimensão do conjunto de dados originais através da correlação entre as diversas variáveis, proporcionando uma melhor visualização das variáveis das amostras em uma dimensão reduzida. Para isso, um novo conjunto de dados, os vetores de base, é construído simultaneamente a um novo conjunto de variáveis linearmente independentes. Esses vetores de base são os novos eixos que correspondem às componentes principais (PC) e, como são gerados de acordo com a variância explicada, o primeiro vetor está ordenado na direção de maior variância (contém maior quantidade de informação). Além de reduzir a dimensão original dos dados, é possível modelar, reconhecer amostras anômalas (chamadas de outliers) e variáveis de maior importância, bem como classificar e prever dados..$^{21,22}$

Dessa forma, é possível realizar uma análise semiquantitativa exploratória da qualidade dos produtos (screening) de forma rápida, com baixo custo operacional e mínimo pré-tratamento da amostra, resultando num procedimento limpo, sem consumo de reagentes e geração de resíduos. ${ }^{14}$

\section{PARTE EXPERIMENTAL}

\section{Materiais escolares}

Neste estudo, foram utilizadas 24 amostras de giz de cera, de 4 marcas diferentes (A, B, C e D), das seguintes cores: azul, verde, amarela, vermelha, laranja e roxa. Também foram usadas 24 amostras de tinta guache (4 marcas das cores: azul, verde, amarela, vermelha, branca e preta). As cores de massa de modelar utilizadas foram azul, verde, amarela, vermelha, laranja e preta, em um total de 21 amostras e 3 marcas. Todas as amostras foram adquiridas em papelarias de Campinas, SP.

Para cada amostra, foram preparadas 3 celas de XRF. As peças de giz de cera e de massa de modelar foram divididas em 3 cilindros de $1 \mathrm{~cm}$ cada, tendo sido polida a base do giz, antes da obtenção do espectro (Figura 1S, material suplementar). Para o guache, cerca de $5 \mathrm{~mL}$ de tinta foram transferidos para cada cela.

\section{Análises dos materiais escolares por XRF}

Para as medidas de XRF, foram utilizadas celas Chemplex 1300, sustentadas por filme de Mylar $^{\circledR}$ (Chemplex 100) com 2,5 $\mu \mathrm{m}$ de espessura. Cada cela foi irradiada em triplicata, obtendo-se 9 espectros por amostra.

O espectrômetro utilizado foi o de XRF por dispersão de energia (EDXRF), Shimadzu EDX700, constituído por um tubo com alvo metálico de $\mathrm{Rh}$ e um detector semicondutor de $\mathrm{Si}(\mathrm{Li})$.

A voltagem aplicada ao tubo de raios $\mathrm{X}$ foi de $50 \mathrm{kV}$, com tempo morto do detector de $25 \%$ e colimador de $5 \mathrm{~mm}$. Os espectros foram obtidos sequencialmente de 0 a $40 \mathrm{keV}$, com resolução de $0,02 \mathrm{keV}$. O tempo de irradiação foi de $850 \mathrm{~s}$, determinado através de estudos prévios de otimização. Para as amostras sólidas (massa de modelar e giz de cera), foi aplicado vácuo, enquanto que para a tinta guache, a obtenção dos espectros foi realizada em pressão ambiente.

Para verificar se havia diferença significativa entre si, os valores de concentração das replicatas de cada elemento químico das amostras foram comparados utilizando-se o teste Q (com nível de confiança de $95 \%) .{ }^{23,24}$ Foi calculada então a média das concentrações, cujos valores foram comparados com as máximas permitidas de metais em brinquedos, uma vez que ainda não existem valores definidos para materiais escolares. ${ }^{11}$

\section{RESULTADOS E DISCUSSÃO}

Para maior clareza na interpretação dos resultados, as amostras foram divididas pelo seu tipo (giz de cera, guache e massa de modelar).

\section{Giz de cera}

Na Figura 1, são apresentados todos os espectros de giz de cera.

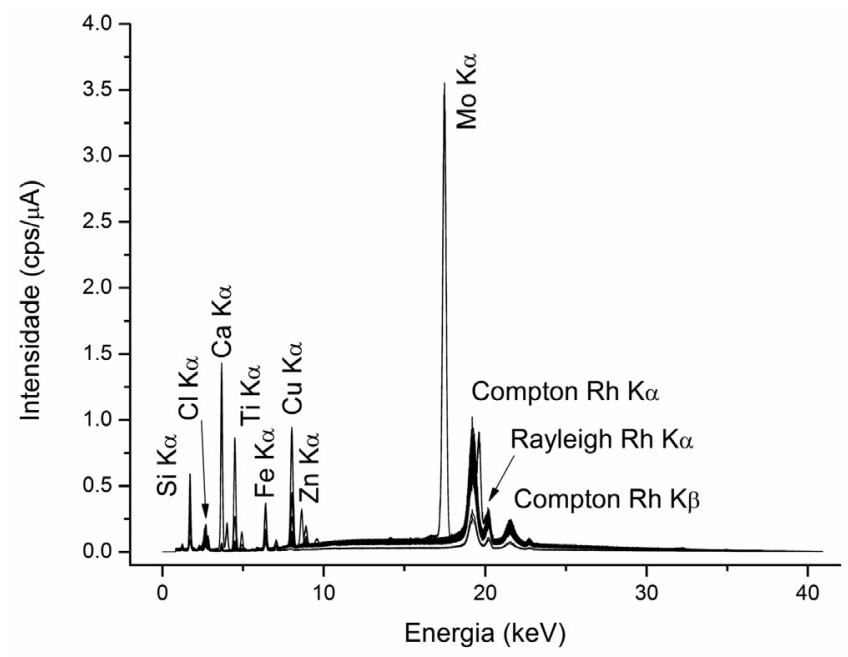

Figura 1. Espectros de XRF para giz de cera de todas as cores das marcas $A, B, C$ e $D$ 
Observa-se na Figura 1 que há semelhança nos perfis dos espectros, estando os elementos $\mathrm{Ca}, \mathrm{Cu}, \mathrm{Fe}, \mathrm{Mg}, \mathrm{S}$ e $\mathrm{Si}$ presentes em todas as amostras, independente de cores ou marcas. As faixas de concentração encontradas estão expostas na Tabela 2.

Tabela 2. Faixas de concentração para os elementos químicos presentes nas amostras de giz de cera $(\mathrm{g} / 100 \mathrm{~g})$

\begin{tabular}{ccc}
\hline \multirow{2}{*}{ Elemento } & \multicolumn{2}{c}{ Faixa de concentração } \\
\cline { 2 - 3 } & Menor valor & Maior valor \\
\hline $\mathrm{Mg}$ & $2,6 \pm 0,4$ & $13 \pm 2$ \\
$\mathrm{Si}$ & $0,97 \pm 0,07$ & $10,6 \pm 0,9$ \\
$\mathrm{~S}$ & $0,045 \pm 0,007$ & $0,085 \pm 0,004$ \\
$\mathrm{Fe}$ & $0,038 \pm 0,002$ & $0,17 \pm 0,02$ \\
$\mathrm{Cu}$ & $0,0051 \pm 0,0007$ & $0,230 \pm 0,009$ \\
$\mathrm{Ca}$ & $0,029 \pm 0,002$ & $3,3 \pm 0,2$ \\
\hline
\end{tabular}

Segundo informações de rótulo dos fabricantes, as matériasprimas dos gizes são ceras, pigmentos orgânicos e carga mineral inerte. Uma composição usual e mais completa é, em massa, de cerca de $40 \%$ de ácidos graxos $\mathrm{C}_{16}-\mathrm{C}_{18}, 15 \%$ de cera microcristalina, $25 \%$ de cera de éster, $15 \%$ de cargas minerais e $5 \%$ de pigmentos. ${ }^{25}$ Cargas minerais normalmente são agregadas com a finalidade de adicionar massa ou modificar propriedades físicas, como opacidade ou viscosidade, por exemplo. Para tanto, devem ter características adequadas com relação ao tamanho de partícula, abrasão, $\mathrm{pH}$ e também deve ser inerte no meio ao qual será adicionada. Compostos como carbonato de cálcio, barita, argila, sílica e talco costumam ser muito utilizados devido ao seu custo e disponibilidade. ${ }^{26}$

A presença dos elementos químicos pode ser atribuída às características das matérias-primas que compõem os gizes. Não apenas cargas, mas pigmentos orgânicos também podem conter metais, como nos complexos metálicos. Nessa categoria, ftalocianinas de cobre apresentam grande importância econômica, fornecendo a maior parte dos pigmentos azuis e verdes utilizados pela indústria. ${ }^{27}$ No início da década de 1990, 25\% dos corantes orgânicos consumidos no mundo pertenciam à classe das ftalocianinas. ${ }^{28}$

Essas informações podem explicar os resultados obtidos, já que as amostras de cor azul apresentaram uma faixa de concentração para o elemento $\mathrm{Cu}(0,094 \pm 0,004$ a 0,230 $\pm 0,009 \mathrm{~g} / 100 \mathrm{~g})$ que é consideravelmente superior à das demais cores, de apenas 0,0051 \pm 0,0007 a $0,037 \pm 0,001 \mathrm{~g} / 100 \mathrm{~g}$. O mesmo ocorreu para amostras de cor verde, que apresentaram faixa também superior às das demais amostras $(0,0165 \pm 0,0004$ a $0,034 \pm 0,004 \mathrm{~g} / 100 \mathrm{~g})$, indicando que o pigmento utilizado pode ser específico para a cor ou uma composição de amarelo com o mesmo empregado nos gizes azuis. ${ }^{29}$

Além do $\mathrm{Cu}$, os elementos $\mathrm{Ca}$, Mo, Ti e $\mathrm{Zn}$ aparecem em concentrações distintas daquelas encontradas na maioria das amostras. Os gizes da marca A e de cor roxa apresentaram uma média de 0,42 $\pm 0,02 \mathrm{~g} / 100 \mathrm{~g}$ para Mo, que não foi detectado em nenhuma outra amostra. Compostos de Mo, na forma de molibdatos, são empregados na indústria como pigmentos, sendo os mais importantes aqueles de cor laranja e vermelha que, associados a outros corantes, podem criar uma grande variedade de cores. ${ }^{29}$ Para os gizes de cor azul da mesma marca, a concentração de Ti foi a maior entre todos, com média de $1,69 \pm 0,05 \mathrm{~g} / 100 \mathrm{~g}$, sendo detectado também nas amostras roxas das marcas A e B e nas verdes da marca B. Todas as amostras da marca B apresentaram teores mais elevados de Ca, sendo a média de 3,1 $\pm 0,2 \mathrm{~g} / 100 \mathrm{~g}$. Por fim, $\mathrm{Zn}$ foi detectado nas amostras vermelhas da marca $\mathrm{D}$, com teor médio de $0,062 \pm 0,003 \mathrm{~g} / 100 \mathrm{~g}$.

Ainda que o número de elementos químicos determinados não seja tão elevado nas amostras, a visualização da presença de elementos em concentrações anômalas para algumas amostras poderia ser mais facilmente obtida através de uma análise exploratória multivariada. Então, foi realizada a Análise de Componentes Principais (PCA), cujos gráficos de scores e loadings para as PC1 X PC2 (Figuras 2 e 3), com 6 componentes principais, permitiram constatar uma evidente separação das amostras roxas da marca $\mathrm{A}$ das demais. Isso ocorreu pela presença do elemento $\mathrm{Mo}\left(\mathrm{K}_{\alpha} 17,4 \mathrm{keV}\right)$ apenas nessas amostras, como indica o gráfico de loadings.

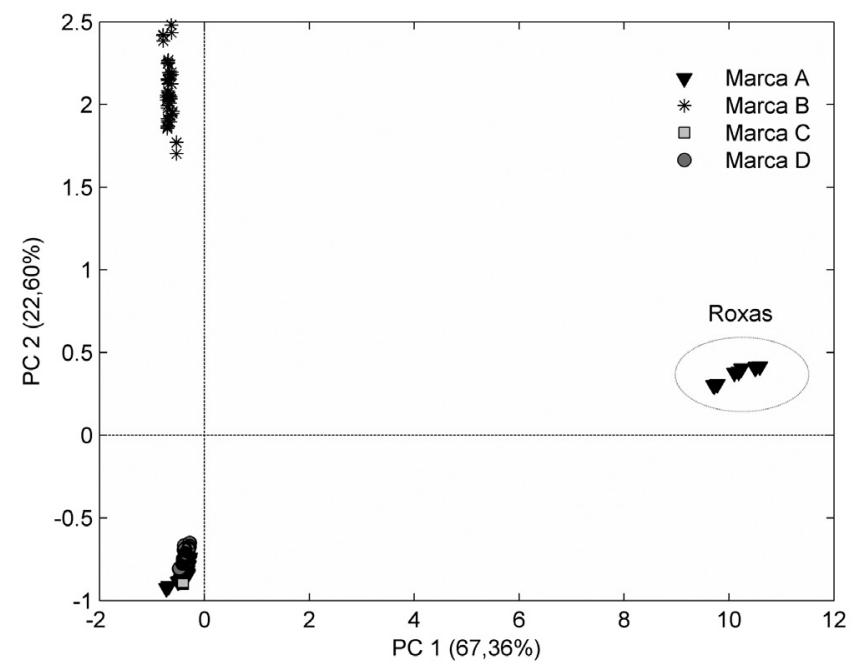

Figura 2. Gráfico de scores das PC1 (67,36\%) x PC2 (22,60\%) para todas as amostras de giz de cera

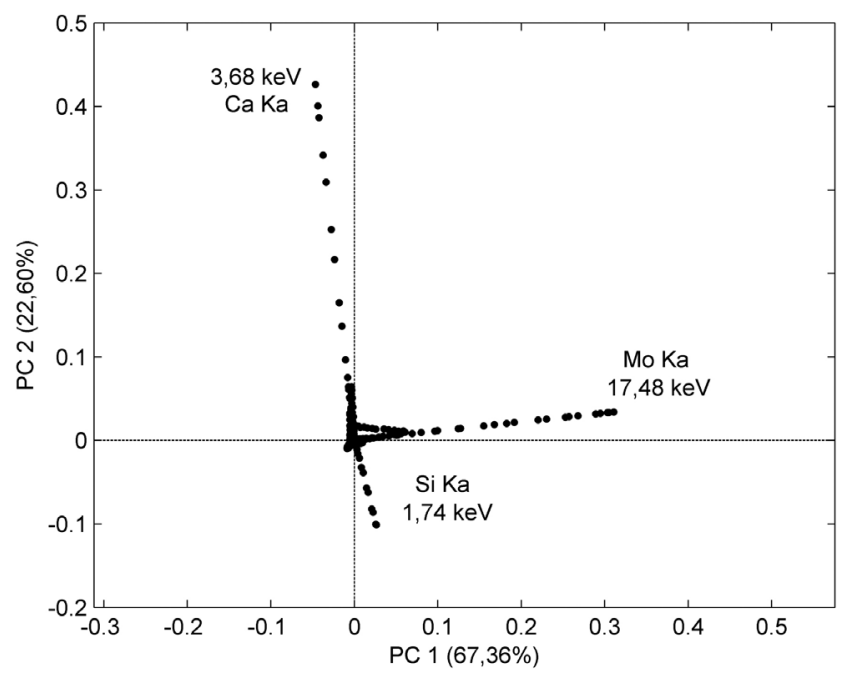

Figura 3. Gráfico de loadings das PC1 (67,36\%) x PC2 (22,60\%) para todas as amostras de giz de cera

Para facilitar a visualização das demais tendências, novo modelo foi realizado excluindo as 9 medidas referentes às amostras roxas da marca A. Pode-se verificar claramente o agrupamento de todas as amostras da marca B pela primeira componente, e uma separação das amostras azuis na segunda componente (Figuras 4 e 5).

A PC1, com 69,36\% da informação do modelo, separa as amostras da marca $\mathrm{B}$ baseadas na presença de $\mathrm{Ca}$, o que pôde ser confirmado ao verificar os loadings na região de $3,69 \mathrm{keV}$, correspondente ao pico K deste elemento. Já a PC2, contendo 12,82\% da informação, foi responsável pela separação das amostras azuis, agrupadas na parte superior do gráfico de scores. Pelo gráfico de loadings, observa-se 


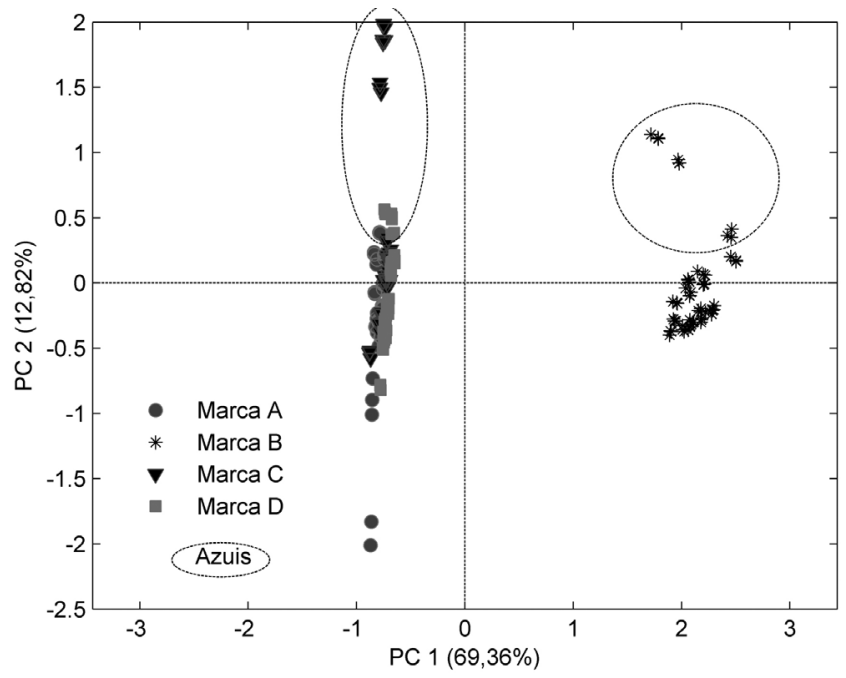

Figura 4. Gráfico de scores das PC1 (69,36\%) x PC2 (12,82\%) sem as amostras de giz de cera roxas da marca A

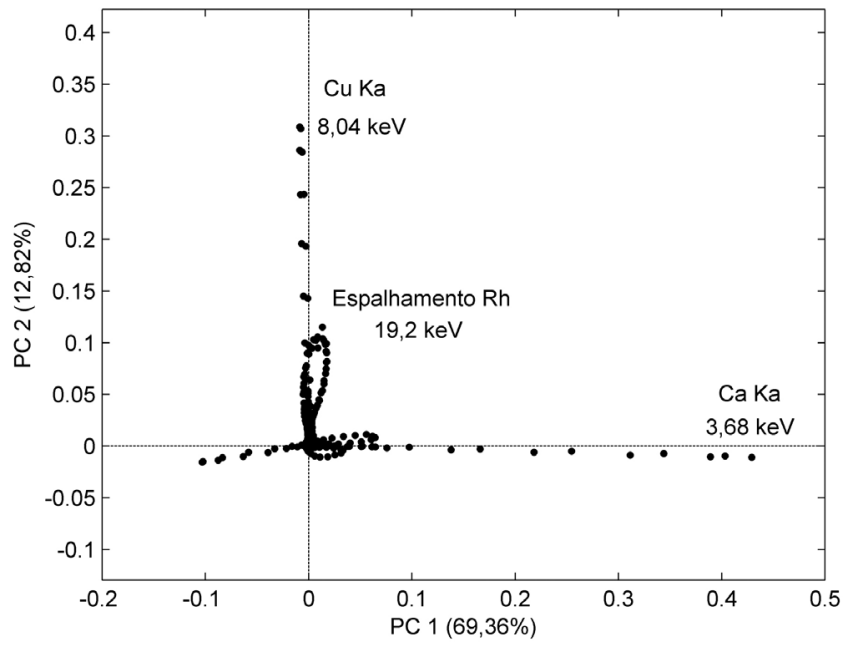

Figura 5. Gráfico de loadings das PC1 (69,36\%) x PC2 (12,82\%) sem as amostras de giz de cera roxas da marca $A$

que o Cu é responsável por essa distinção (8,04 keV). Analisando-se as PC3 e PC4 (Figura 6), com 7,91 e 6,52\% de informação, respectivamente, há uma orientação das amostras azuis da marca A e das roxas da marca B para o quadrante superior direito. Esta tendência é função da presença de Ti, conforme pode ser comprovado pelos valores significativos de energia referentes ao pico $\mathrm{K}$ do elemento, de $4,50 \mathrm{keV}$ (Figura 7). No mesmo gráfico pode-se observar uma influência do efeito de espalhamento de raios $\mathrm{X}$, que orienta as amostras no sentido do quadrante diagonal superior à esquerda. Isso ocorreu devido aos compostos orgânicos presentes nas amostras, como as ceras e os pigmentos.

A PC5, com 1,28\% de informação, permite verificar um agrupamento das amostras da marca $\mathrm{C}$ devido ao espalhamento (aproximadamente $19 \mathrm{keV})$ e a presença de $\mathrm{Cl}(2,62 \mathrm{keV})$. Simultaneamente, a PC6, com menos de 1\% de informação, separa as amostras vermelhas da marca $\mathrm{D}$ por conter o elemento $\mathrm{Zn}(\mathrm{K} 8,63 \mathrm{keV})$, conforme pode ser visto nos gráficos de scores e de loadings (Figura $2 \mathrm{~S}$, material suplementar).

Apenas uma única amostra apresentou nível preocupante para elementos químicos controlados pela norma. Trata-se da amostra de cor vermelha, da marca $\mathrm{D}$, cujo teor de bário encontrado foi aproximadamente $38 \%$ superior ao permitido (Tabela 1 ).

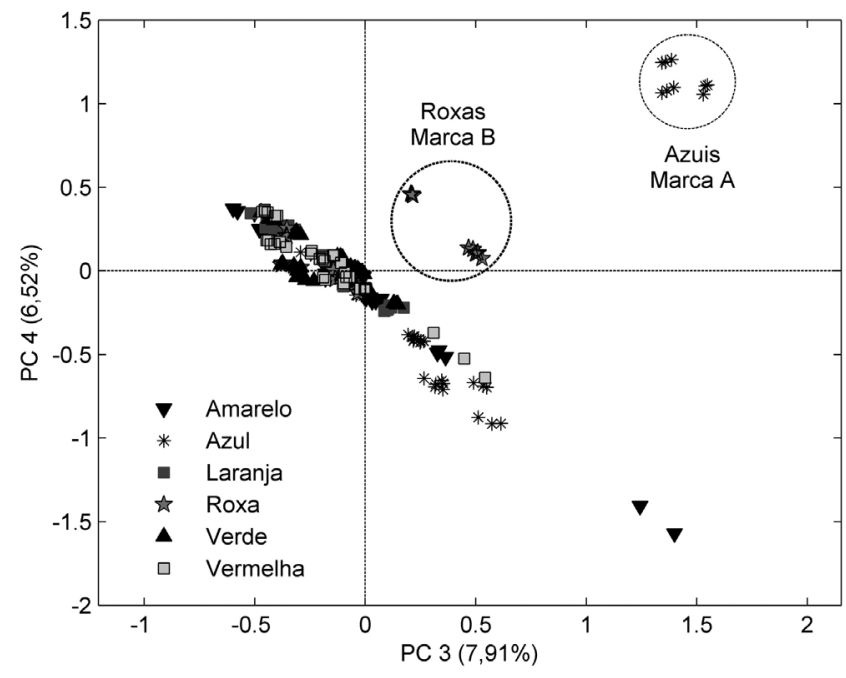

Figura 6. Gráfico de scores das PC3 (7,91\%) x PC4 (6,52\%) sem as amostras de giz de cera roxas da marca $A$

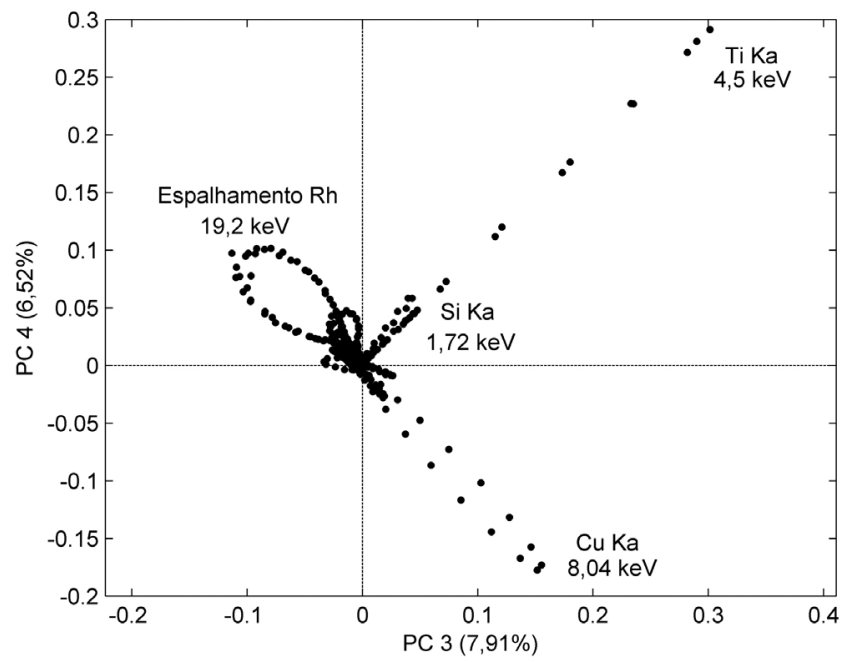

Figura 7. Gráfico de loadings das PC3 (7,91\%) x PC4 (6,52\%) sem as amostras de giz de cera roxas da marca $A$

\section{Tinta guache}

A análise dos espectros de todas as amostras de guache (Figura 3S, material suplementar) mostra que a composição destes materiais também não varia entre as marcas, sendo que $\mathrm{Br}, \mathrm{Ca}, \mathrm{Cu}, \mathrm{Fe}, \mathrm{Nb}, \mathrm{Sr}$, Ti e $\mathrm{Zr}$ são elementos comuns a todas elas.

As faixas de concentração para os elementos químicos nas amostras podem ser vistas na Tabela 3 e a justificativa da presença dos elementos químicos é dada pelas circunstâncias explicadas anteriormente, uma vez que essas tintas também possuem elementos como cargas inertes e pigmentos. Segundo informações de rótulo dos fabricantes, guaches contêm resina, pigmento, agente espessante, carga inerte, conservante do tipo benzotiazol e água.

Os resultados da PCA facilitam o reconhecimento de tendências mais específicas quanto à presença dos elementos químicos. O modelo foi construído com todos os espectros das amostras centrados na média e considerando 6 PCs (99,76\% da variância explicada).

O gráfico de PC1 X PC2 (Figura 4S, material suplementar) mostra uma tendência de agrupamento das amostras por marca, devido ao elemento $\mathrm{Ca}(3,69 \mathrm{keV})$, que provavelmente é proveniente de carga mineral. ${ }^{26} \mathrm{~A}$ concentração do elemento é maior para a marca $\mathrm{D}$, seguida pelas C, A e B, respectivamente. 
Tabela 3. Faixas de concentração obtidas para as diversas amostras de tinta guache $(\mathrm{g} / 100 \mathrm{~g})$

\begin{tabular}{ccc}
\hline \multirow{2}{*}{ Elemento } & \multicolumn{2}{c}{ Faixa de concentração } \\
\cline { 2 - 3 } & Menor valor & Maior valor \\
\hline $\mathrm{Br}$ & $0,0024 \pm 0,0001$ & $0,0137 \pm 0,0002$ \\
$\mathrm{Ca}$ & $0,011 \pm 0,002$ & $10,08 \pm 0,02$ \\
$\mathrm{Cu}$ & $0,0013 \pm 0,0003$ & $0,0827 \pm 0,0004$ \\
$\mathrm{Fe}$ & $0,0039 \pm 0,0006$ & $0,0549 \pm 0,0006$ \\
$\mathrm{Nb}$ & $0,0012 \pm 0,0001$ & $0,0019 \pm 0,0001$ \\
$\mathrm{Sr}$ & $0,0015 \pm 0,0002$ & $0,0412 \pm 0,0003$ \\
$\mathrm{Ti}$ & $0,094 \pm 0,001$ & $5,403 \pm 0,009$ \\
$\mathrm{Zr}$ & $0,0012 \pm 0,0001$ & $0,0308 \pm 0,0003$ \\
\hline
\end{tabular}

Nesta mesma Figura 4S, material suplementar, verifica-se que na PC2 (28,13\% da variância), as amostras se separam em virtude do teor de Ti $(4,50 \mathrm{keV})$, o que justifica a discriminação das tintas brancas, principalmente para a marca A. É importante salientar que o $\mathrm{TiO}_{2}$ é o pigmento branco mais empregado na indústria. ${ }^{27}$

O gráfico de PC3 x PC4 (Figura 5S, material suplementar) indica uma forte correlação do elemento $\mathrm{Cu}(\mathrm{K} \mathrm{8,04} \mathrm{keV})$ na terceira componente, com o agrupamento das amostras de cores azul e verde, devido aos corantes utilizados. ${ }^{28}$ Já a PC4 mostra uma influência positiva para o elemento $\mathrm{Sr}(\mathrm{K} 14,14 \mathrm{keV})$ e negativa para o elemento $\mathrm{Fe}(\mathrm{K} 6,39 \mathrm{keV})$. Com relação às amostras, nota-se que as da marca D são aquelas com maior teor de $\mathrm{Sr}$ e as da marca $\mathrm{C}$, de Fe. Além disso, é interessante notar a influência do pico de energia $19,2 \mathrm{keV}$, que orienta as amostras no plano em função do fenômeno de espalhamento da fonte de raios X. No caso específico, o alvo metálico do tubo de raios $\mathrm{X}$ do espectrômetro utilizado é de $\mathrm{Rh}$ e o seu K, 20,21 keV e o espalhamento ocorre na região entre 19 a 22 $\mathrm{keV}$. Esse comportamento é tão mais pronunciado quanto maior for o teor de compostos orgânicos nas amostras, o que é esperado nos guaches devido aos conservantes, resinas e água. Por fim, também foram avaliadas as quintas e sextas componentes (Figura 6S, material suplementar), para verificar possíveis tendências de agrupamento das amostras. No caso da PC5, com $0,25 \%$ de informação, o grande peso é dado pelo espalhamento da fonte de raios X (K 19,2 keV) e para os elementos $\mathrm{Sr}$ (K 14,14 keV) e $\mathrm{Cu}(\mathrm{K} 8,04 \mathrm{keV})$, que separam as amostras das marcas A e $\mathrm{C}$ no quadrante direito e, principalmente, as da marca $\mathrm{D}$, no esquerdo. Mais importante que isso, a porcentagem da informação contida na PC6, de apenas $0,08 \%$, poderia ser interpretada erroneamente como somente ruído sendo modelado. Porém, os loadings dessa PC indicam grande influência do pico K do elemento $\mathrm{Br}(11,6 \mathrm{keV})$ e o gráfico de scores mostra o agrupamento das amostras da marca D no sentido contrário, mostrando que essas amostras não possuem o elemento em sua composição.

Essa é a informação mais importante com relação a elementos químicos tóxicos, uma vez que a sobredosagem de compostos derivados de $\mathrm{Br}$ pode induzir a um funcionamento inadequado do organismo. Os valores máximos para $\mathrm{Br}$ nas tintas guache foram encontrados nas amostras da marca A $(0,0137 \pm 0,0002 \mathrm{~g} / 100 \mathrm{~g})$. Como não se tem informação do limite máximo permitido para este, a comparação não pôde ser realizada. Para os demais elementos químicos contemplados, todas as amostras de guache estão em acordo com os valores descritos.

\section{Massa de modelar}

A análise dos espectros das amostras de massa de modelar
(Figura 7S, material suplementar) indica que a composição das massas, que contêm ceras, cargas inertes e pigmentos, segundo os rótulos, não varia consideravelmente. Essa semelhança nos espectros se reflete pela presença de $\mathrm{Ca}, \mathrm{Fe}, \mathrm{Mg}$, $\mathrm{Si}$ e $\mathrm{Sr}$ em todas as amostras, provavelmente componentes das cargas minerais empregadas. Na Tabela 4, estão expostas as faixas de concentração para os elementos químicos.

Tabela 4. Faixas de concentração obtidas para as amostras de massa de modelar $(\mathrm{g} / 100 \mathrm{~g})$

\begin{tabular}{ccc}
\hline \multirow{2}{*}{ Elemento } & \multicolumn{2}{c}{ Faixa de concentração } \\
\cline { 2 - 3 } & Menor valor & Maior valor \\
\hline $\mathrm{Ca}$ & $20 \pm 1$ & $27 \pm 1$ \\
$\mathrm{Fe}$ & $0,012 \pm 0,002$ & $0,62 \pm 0,03$ \\
$\mathrm{Mg}$ & $2,5 \pm 0,3$ & $5 \pm 1$ \\
$\mathrm{Si}$ & $0,13 \pm 0,02$ & $0,65 \pm 0,03$ \\
$\mathrm{Sr}$ & $0,007 \pm 0,001$ & $0,009 \pm 0,001$ \\
\hline
\end{tabular}

Um caso interessante é o do $\mathrm{Fe}$, que nas amostras pretas da marca A apresentou concentração muito superior $(0,62 \pm 0,03 \mathrm{~g} / 100 \mathrm{~g})$ à média das amostras, de $0,017 \pm 0,004 \mathrm{~g} / 100 \mathrm{~g}$. Isso indica que um corante inorgânico do tipo óxido de ferro preto pode ter sido empregado para colorir o material..$^{29}$ Comportamento análogo é observado para o Ti, presente apenas nas amostras brancas das marcas A e C, o que permite inferir que $\mathrm{TiO}_{2}$ deve ter sido empregado para colorir, além de garantir outras características como opacidade. ${ }^{29}$ No caso da marca B, como nenhum dos dois elementos químicos mais empregados como pigmento branco, Ti e $\mathrm{Zn}$, foram detectados, um corante orgânico pode ter sido empregado. ${ }^{30}$ Não foi observado para as massas de modelar comportamento similar das amostras de guache e giz de cera com relação à presença de $\mathrm{Cu}$ nas amostras azuis. Isso indica que outro tipo de corante deve estar sendo empregado. Em amostras verdes de duas marcas foi encontrado $\mathrm{Cu}$, mostrando a tendência de escolha de pigmento discutida anteriormente. ${ }^{28}$

Os resultados da análise multivariada confirmam as explicações obtidas pela observação dos espectros e das concentrações calculadas pelo método de parâmetros fundamentais (PF). Com apenas $6 \mathrm{PCs}$ foi possível explicar 99,20\% da variância do modelo, sendo que na PC1 $(74,76 \%)$ há uma tendência de separação em função do teor de $\mathrm{Ca}$, o que se justifica pelo fato do elemento ser aquele com as maiores concentrações. A PC2 (17,29\%) também faz referência ao Ca (3,69 keV), porém não há tendência de agrupamento para as amostras (Figura $8 \mathrm{~S}$, material suplementar). O comportamento observado na PC2 se repete nas PC4 e PC5, que sofrem ainda influência do espalhamento de raios X, mas não explicam a composição elementar das massas e, por isso, os gráficos não são mostrados neste trabalho.

Comportamento diferente foi observado nas PC3 e PC6 que, mesmo com uma porcentagem menor de informação (4,74 e 0,32\%, respectivamente), mostram tendências de agrupamento. Na PC3, a influência é devida ao pico $\mathrm{K}$ do $\mathrm{Fe}(6,39 \mathrm{keV})$ e, assim, as amostras de cor preta e da marca A são separadas das demais, devido à concentração superior deste elemento. O mesmo ocorre na PC6 para as amostras brancas das marcas $\mathrm{A}$ e $\mathrm{C}$, que possuem maiores teores de Ti; o que pode ser conferido nos gráficos de scores e de loadings (Figura 9S, material suplementar).

Por fim, não foram encontrados elementos químicos tóxicos e nem concentrações de elementos em desacordo com a norma do INMETRO para brinquedos; o que permite afirmar que as amostras não representam um risco direto à segurança das crianças no que se refere à contaminação por metais. 


\section{CONCLUSÕES}

O estudo realizado permitiu constatar que o método proposto envolvendo XRF é adequado para investigar, de forma semiquantitativa, a presença de elementos inorgânicos tóxicos em amostras de tinta guache, massa de modelar e giz de cera, sendo um procedimento rápido, multielementar e que atende as especificações da Química Verde.

A escolha destas amostras, que podem ser enquadradas tanto como brinquedos como material escolar, deve-se ao fato de serem produtos de grande apelo infantil, podendo causar danos à saúde das crianças.

Apesar da maioria das amostras não apresentar indícios de promoverem contaminação por conta da presença de elementos químicos tóxicos, cabe ressaltar que em três marcas de guache estudadas foi identificada a presença de $\mathrm{Br}$ como elemento químico potencialmente tóxico, enquanto teores elevados de bário foram encontrados em uma amostra de giz de cera. Tais informações permitem afirmar a necessidade de estudos mais detalhados, assim como fiscalizações mais rígidas por parte dos órgãos oficiais.

\section{MATERIAL SUPLEMENTAR}

O esquema de corte das amostras de giz de cera e massa de modelar (Figura 1S), os espectros das tintas guache (Figura 3S) e das massas de modelar (Figura 7S) e os gráficos de scores e loadings para os modelos de PCA construídos (Figuras 2S, 4S, 5S, 6S, 8S e 9S) possuem acesso livre, na forma de arquivo PDF, em http:// quimicanova.sbq.org.br.

\section{AGRADECIMENTOS}

Às agências de fomento Capes, CNPq e FAPESP pelo apoio financeiro.

\section{REFERÊNCIAS}

1. Carvalho, F. M.; Silvany-Neto, A. M.; Lima, M. E. C.; Tavares, T. M.; Azaro, M. G. A.; Quaglia, G. M. C.; Rev. Saúde Pública 1987, 21, 44.

2. Moreira, F. R.; Moreira, J. C.; Rev. Panam. Salud Publica 2004, 15, 119.

3. Lynch, R. A.; Boatright, D. T.; Moss, S. K.; Publ. Health Rep. 2000 , $115,537$.

4. Zietz, B.; de-Vergara, J. D.; Kevekordes, S.; Dunkelberg, H.; Sci. Total. Environ. 2001, 275, 19.

5. VanArsdale, J. L.; Leiker, R. D.; Kohn, M.; Merritt, T. A.; Horowitz, Z.; Pediatrics 2004, 114, 1096.

6. Kumar, A.; Pastore, P.; Curr. Sci. 2007, 93, 818.

7. Zini, J.; Ferreira, J. C.; Carvalho, F. M. S.; Bustillos, J. O. W. V.; Scapin, M. A.; Salvador, V. L. R.; Abrão, A.; Quim. Nova 2009, 32, 833.

8. Corazza, M.; Baldo, F.; Pagnoni, A.; Miscioscia, R.; Virgili, A.; Acta Dermatol. Venereol. 2009, 89, 130.

9. Godoi, Q.; Santos Jr., D.; Nunes, L. C.; Leme, F. O.; Rufini, I. A.; Agnelli, J. A. M.; Trevizan, L. C.; Krug, F. J.; Spectrochim. Acta, Part B 2009, 64, 573 .
10. http://www.inmetro.gov.br/imprensa/releases/brinquedo2.asp, acessada em Março 2013.

11. http://www.inmetro.gov.br/consumidor/produtos/brinquedos2.asp, acessada em Março 2013

12. http://www.inmetro.gov.br/imprensa/releases/artigos-escolares-Jul2010.pdf, acessada em Março 2013.

13. Inczédy, J.; Lengyel, T.; Ure, A. M.; Compendium of Analytical Nomenclature: Definitive Rules, $3^{\text {rd }}$ ed., International Union of Pure and Applied Chemistry: Oxford, 1998.

14. Jenkins, R.; De Vries, J. L.; Practical X-Ray Spectrometry, $2^{\text {nd }}$ ed., Springer: New York, 1970.

15. Farquharson, M. J.; Geraki, K.; X-Ray Spectrom. 2004, 33, 240.

16. Schimidt, F.; Bueno, M. I. M. S.; Poppi, R. J.; Quim. Nova 2002, 25, 949.

17. Bode, P.; Bueno, M. I. M. S.; Bortoleto, G. G.; Hoffmann, G.; van den Ingh, T. S. G. A. M.; Rothuizen, J.; Anal. Bioanal. Chem. 2008, 390, 1653.

18. Calza, C.; Anjos, M. J.; Bueno, M. I. M. S.; Souza, S. M.; Brancaglion Jr, A.; Lima, T. A.; Lopes, R. T.; X-Ray Spectrom. 2007, 36, 348.

19. Da-Col, J. A.; Bueno, M. I. M. S.; Quim. Nova 2009, 32, 2407.

20. Schwab, N. V.; Da-Col, J. A.; Terra, J.; Bueno, M. I. M. S.; J. Braz. Chem. Soc. 2012, 23, 546.

21. Geladi, P.; Spectrochim. Acta, Part B 2003, 58, 767.

22. Wold, S.; Esbensen, K.; Geladi, P.; Chemom. Intell. Lab. Syst. 1987, 2 , 37.

23. Baccan, N.; Andrade, J. C.; Godinho, O. E. S.; Barone, J. S.; Química Analítica Quantitativa Elementar, $3^{\mathrm{a}}$ ed., Edgard Blücher: São Paulo, 2001.

24. Miller, J. N.; Miller, J. C.; Statistics and Chemometrics for Analytical Chemistry, Pearson Education: Harlow, 2000.

25. Colditz, M.; Kunkel, E.; Bohne, K. H.; Drawing and Writing Materials. Ullmann's Encyclopedia of Industrial Chemistry, Wiley-VCH: Weinheim, 2000, vol. 11

26. Hewitt, D. F.; Mineral Fillers. Industrial Mineral Report 41, Ontario Geological Survey: Ontario, 1973.

27. Chen, X.; Mao, S. S.; Chem. Rev. 2007, 107, 2891.

28. Löbbert, G.; Phthalocyanines. Ullmann's Encyclopedia of Industrial Chemistry, Wiley-VCH: Weinheim, 2000, vol. 27.

29. Völz, H .G.; Pigments, Inorganic, 1. General. Ullmann's Encyclopedia of Industrial Chemistry, Wiley-VCH: Weinheim, 2009, vol. 27; Buxbaum, G.; Printzen, H.; Mansmann, M.; Räde, D.; Trenczek, G.; Wilhelm, V.; Schwarz, S.; Wienand, H.; Adel, J.; Adrian, G.; Brandt, K.; Cork, W. B.; Winkeler, H.; Mayer, W.; Schneider, K.; Pigments, Inorganic, 3. Colored Pigments. Ullmann's Encyclopedia of Industrial Chemistry, Wiley-VCH: Weinheim, 2009, vol. 27.

30. Auer, G.; Woditsch, P.; Westerhaus, A.; Kischkewitz, J.; Griebler, W.; Liedekerke, M.; Pigments, Inorganic, 2. White Pigments. Ullmann's Encyclopedia of Industrial Chemistry, Wiley-VCH: Weinheim, 2009, vol. 27. 


\section{ANÁLISE EXPLORATÓRIA RÁPIDA E NÃO DESTRUTIVA (SCREENING) DA PRESENÇA DE ELEMENTOS QUÍMICOS TÓXICOS EM MATERIAL ESCOLAR POR FLUORESCÊNCIA DE RAIOS X}

José Augusto Da-Col, Rafael Ortiz Sanchez, Juliana Terra e Maria Izabel Maretti Silveira Bueno* Instituto de Química, Universidade Estadual de Campinas, CP 6154, 13084-971 Campinas - SP, Brasil

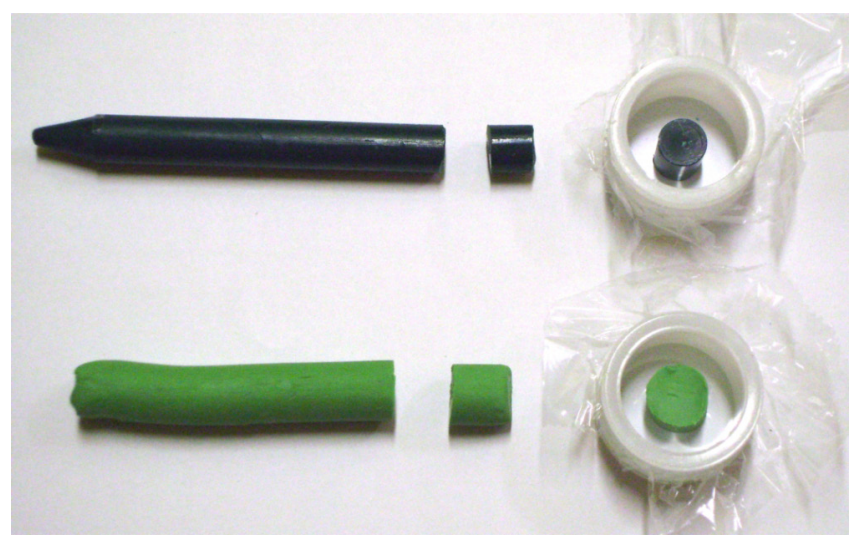

Figura 1S. Esquema de corte das amostras de giz de cera (amostra superior) e massa de modelar para acomodação na cela de análise do espectrômetro de XRF
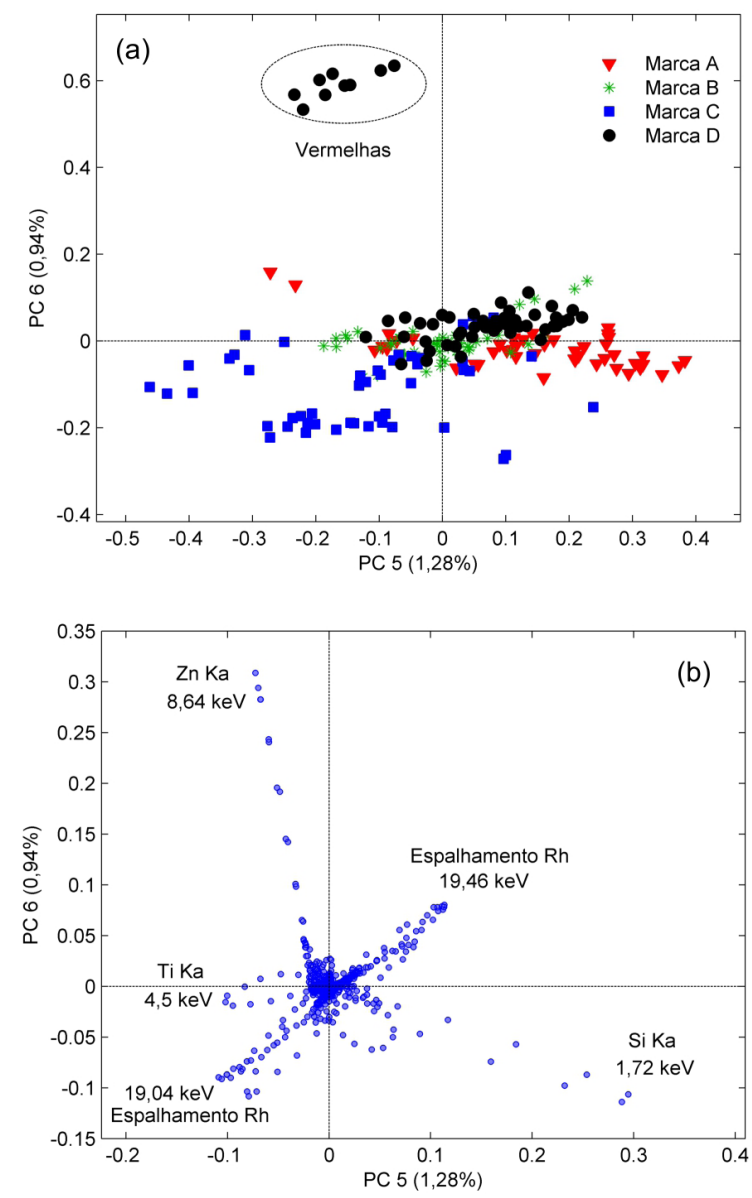

Figura 2S. Gráficos de scores e loadings das PC5 (1,28\%) x PC6 (0,94\%) sem as amostras de giz de cera roxas da marca $A$

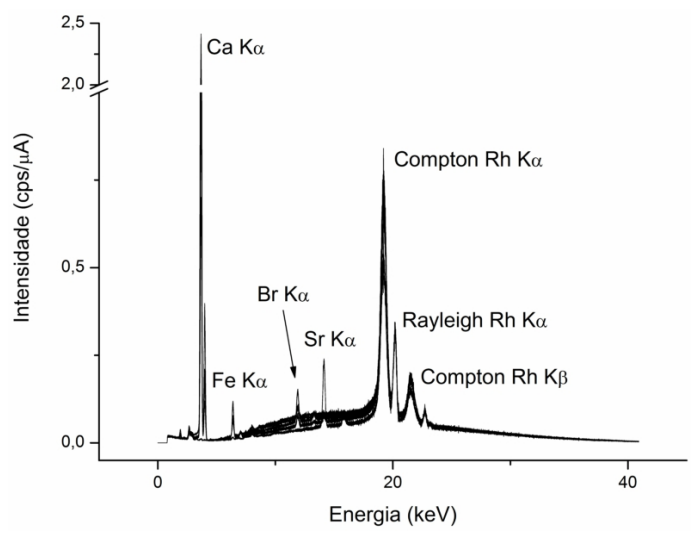

Figura 3S. Espectros de XRF para o conjunto completo das 4 marcas e 6 cores das tintas guache
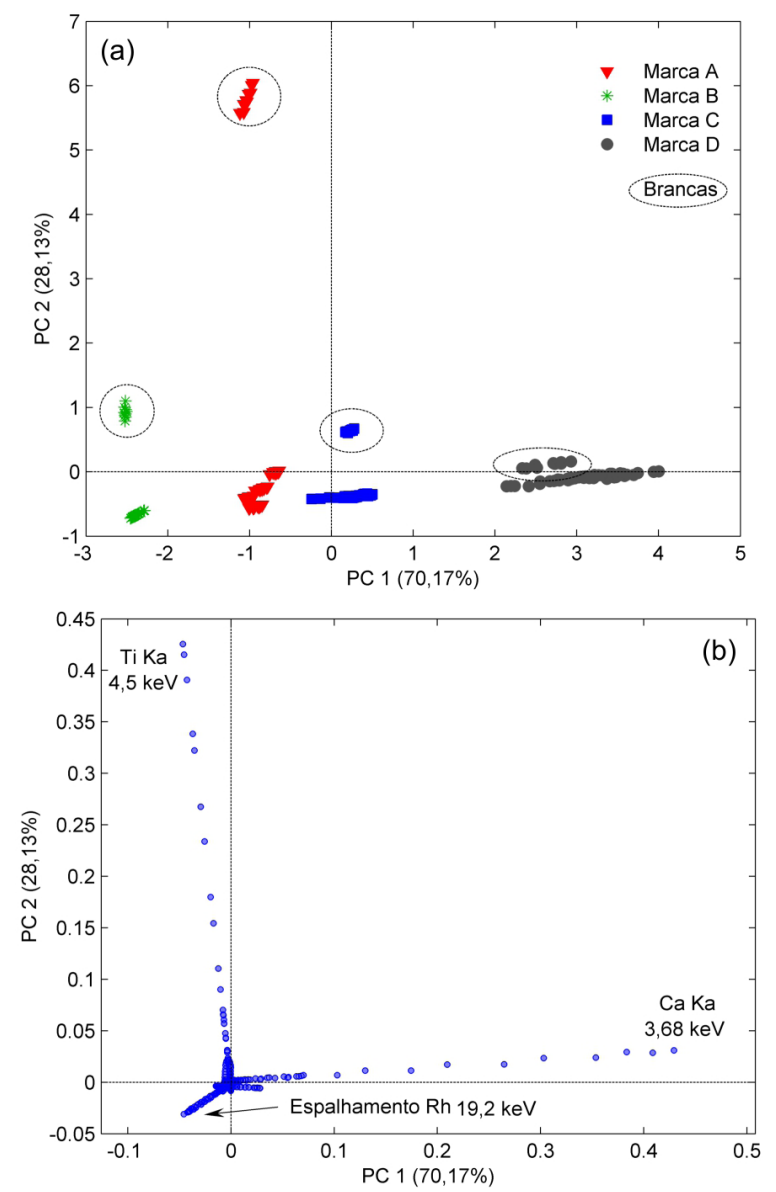

Figura 4S. Gráficos de scores e loadings das PC1 (70,17\%) x PC2 (28,13\%) para as amostras de tinta guache 

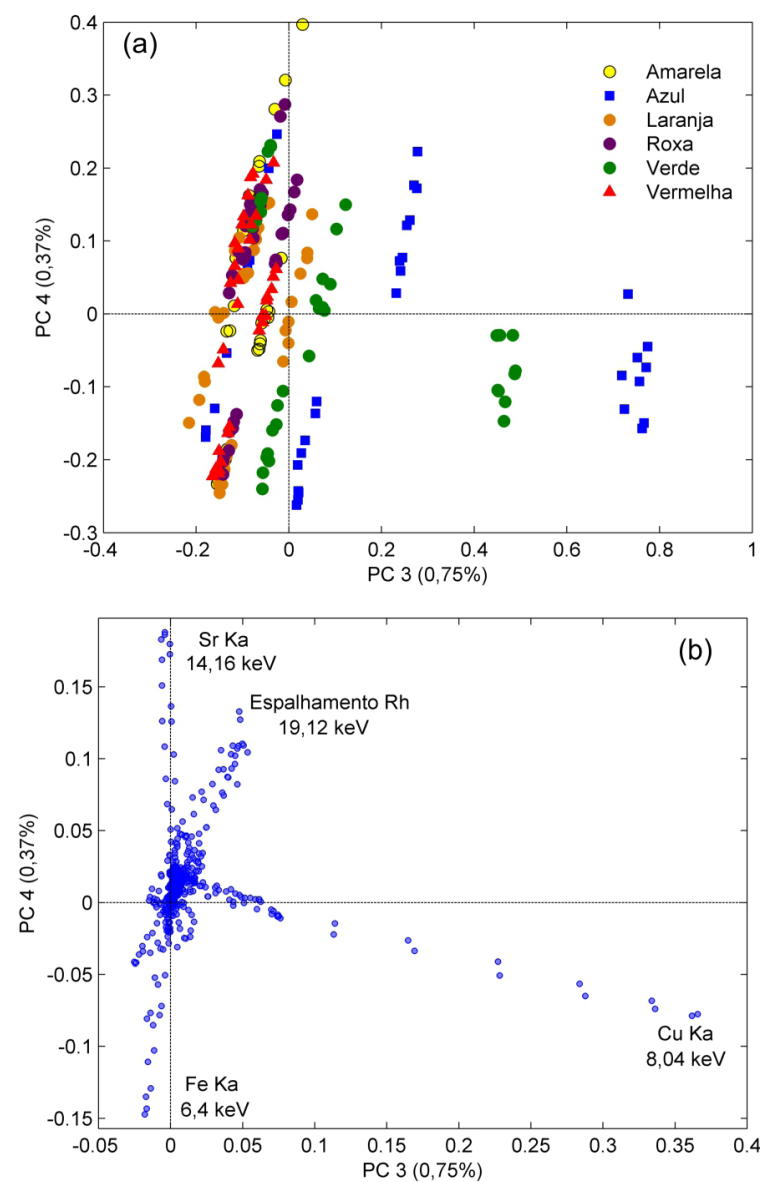

Figura 5S. Gráficos de scores e loadings das PC3 (0,75\%) x PC4 (0,37\%) para as amostras de tinta guache
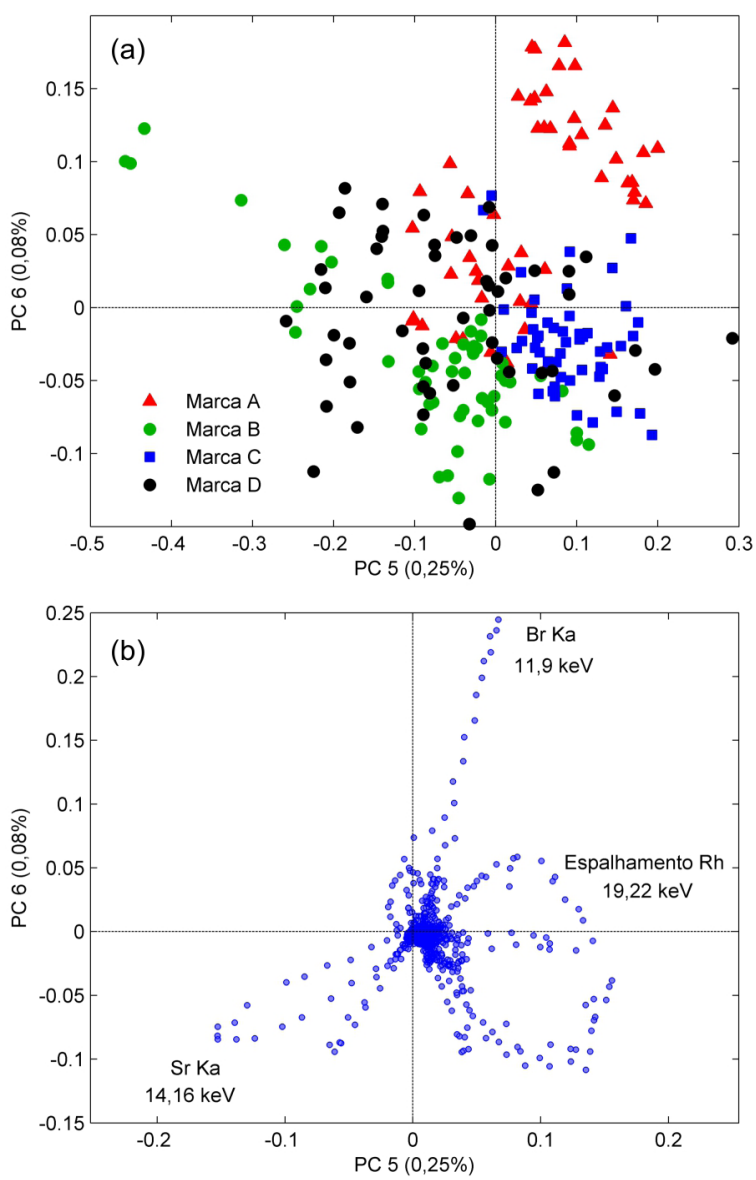

Figura 6S. Gráficos de scores e loadings das PC5 (0,25\%) x PC6 (0,08\%) para as amostras de tinta guache

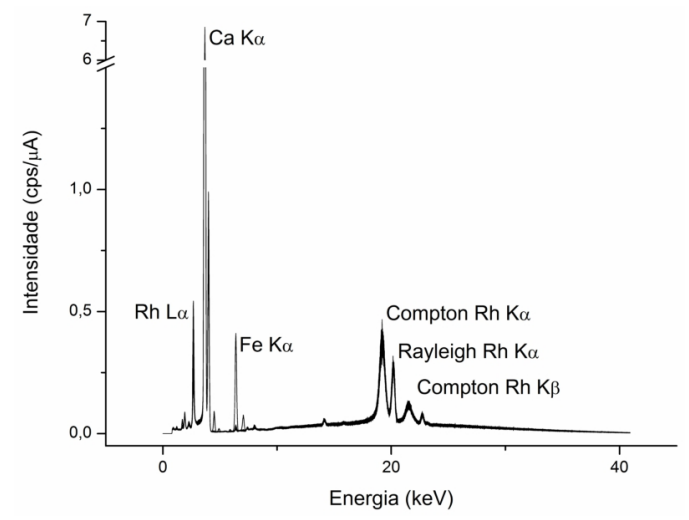

Figura 7S. Espectros de XRF para o conjunto completo das 3 marcas e 7 cores de massa de modelar 

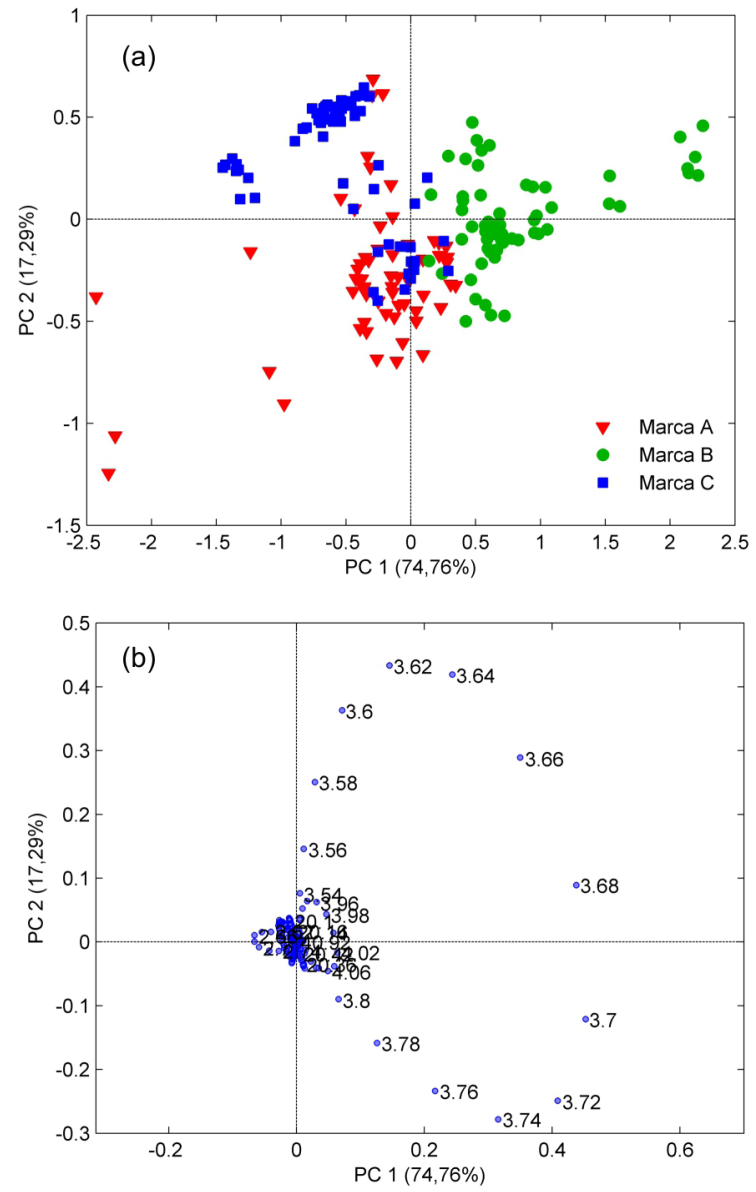

Figura 8S. Gráficos de scores e loadings das PC1 (74,76\%) x PC2 (17,29\%) para as amostras de massa de modelar
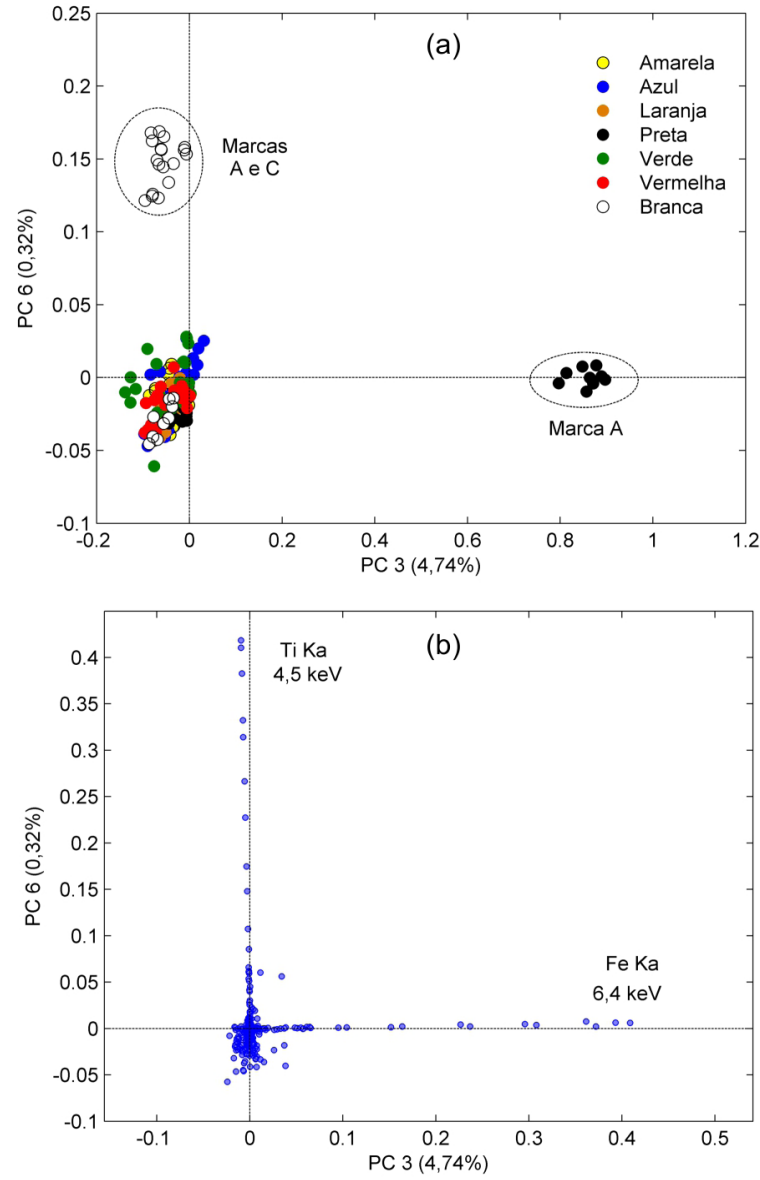

Figura 9S. Gráficos de scores e loadings das PC3 (4,74\%) x PC6 (0,32\%) para as amostras de massa de modelar 